\title{
Time-Delay Compensation in Environment Construction Using Laser Range Finder
}

\author{
Hany Nasry, Chunlan Ye, Jianwei Gong, and Huiyan Chen
}

\begin{abstract}
Teleoperation is operating at a distance and thus extending human capabilities to performing tasks remotely by providing the operator with similar conditions as those at the remote location. Generally, operator should be supported in real-time with an accurate data about the teleoperation environment. This paper proposes a time-delay compensation method for real-time environment construction for vehicle teleoperation as a part of the work for modifying the well-developed Autonomous Ground Vehicle of Beijing Institute of Technology (BIT AGV) to be teleoperated using the Laser scanner data. The proposed Time-Delay Compensation Algorithm includes aprediction process of the vehicle position in the future time and an environment construction w. $r$. t. the predicted position when the vehicle is still in the current time. Results of the real and simulation experiments for the algorithm illustrate the simplicity in calculations, performance and effectiveness of the algorithm to compensate time-delay in environment construction for Vehicle Teleoperation.
\end{abstract}

Index Terms-Environment construction, laser scanner teleoperation, time-delay.

\section{INTRODUCTION}

Teleoperation originates in the mid-1940s, when Goertz built the first mechanically controlled master-slave teleoperator. During the early 1960s an increased interest and several experiments are done to understand the effects of delay in teleoperation (Ferrell, 1965; Sheridan and Ferrell, 1963). Teleoperated ground vehicles are classified into three categories: exploration rovers, Unmanned Ground Vehicles (UGV), and hazardous duty. From the historical researches there are several complications arise in the teleoperated systems since the communication medium contributes to the complexity of the system and introduces distortion, delays, and losses that hinder stability and performance particularly for low-bandwidth, high-delay applications. And as a result of the past researches, the main goals of teleoperation are: Stability and Telepresence of the system. These issues have motivated the theoretic research in teleoperation over the past decades [1].

Teleoperation is considered as a one level from the autonomy Levels which are: Teleoperation with direct remote control; in which there is a visual contact between the operator (the master) and the robot (the slave) and no sensor feedback is needed and Teleoperation with indirect visual feedback; where the operator in the Ground Control Station

Manuscript received November 10, 2012; revised February 1, 2013.

Hany Nasry, Chunlan Ye, and Jianwei Gong are with the Intelligent Vehicle Research Center, BIT, Beijing, China (e-mail: haninzb@yahoo.com, lanyespring@126.com, gongjianwei@bit.edu.cn).

Huiyan Chen is with the head of the Intelligent Vehicle Research Center and Professor in the School of Mechanics, BIT, Beijing, China (e-mail: Chen_h_y@263.net).
(GCS) depends on sensor data to control the robot, Semi-autonomous (supervisory) control; in this level there is a shared control between the operator and the robot. Finally, Autonomous robots; where the robot performs the required tasks autonomously with no operator interference [2]. In vehicle teleoperation, the control levels are divided into: Low Level, Semi-autonomous, and High Level (Autonomous) teleoperation. On the high level, the vehicle relieves the user from significant workload; the operator just gives the vehicle a goal point and the vehicle autonomously drives to the goal. If the vehicle functionality is not fully autonomous, the user can use a lower level of autonomy, which are semi-autonomous or direct control (driving), to solve a task. In semi-autonomous level, it is a trade control between the GCS and the vehicle as the operator sends key way-points along which the vehicle moves depend on its own skills. The lowest level teleoperation is the wheel-drive control, where the operator directly controls or drives the vehicle from the GCS and this is the hardest level for the operator.

In autonomous mobile, vehicles typically make use only of information they can acquire through their sensors to execute certain actions and controls depending on these data. For an example of these actions are; path following, obstacle avoidance, speed control, automatic steering control, traffic lights recognition, preceding vehicle detection and tacking. In lower levels teleoperation, the situation is different because all these sensors data are first fused together and then sent to the ground control station (GCS). Because the operator and the vehicle are located at different sites, time-delays present in the communication channels. As a result, the data received to the GCS is not accurate and out-of-date which hinders the teleoperation process [3],[4]. To solve this problem, this paper proposes an algorithm to compensate time-delay in environment construction for vehicle teleoperation using Lidar data only.

The paper is organized as follows; the second section presents the time-delay problems and their negative effect in teleoperation. In the third section, we explain the time-delay compensation algorithm, in which we explain the prediction process of the vehicle position in the future position. And, by knowing the vehicle current velocity and heading angle, the 2-D static environment is constructed w. r. t. the vehicle predicted position using the laser scanner data in the current time. The experimental and the simulation results are presented in the fourth section. Finally, conclusion and proposed future work of our algorithm are shown in the fifth section.

\section{TELEOPERATION PROBLEMS}

In teleoperation, generally a direct or indirect visual 
feedback should be available. In direct visual feedback there is no problem as the time-delay can be neglected. But for indirect visual feedback, teleoperation is achieved using many sensors. These sensors are subjected to data transmission time-delay, fusion complexities and stability problems. For existing distance between the local and remote environments, or the transmission is performed using a "slow" communication channel, time-delay appears. So, the user should wait to get the actual results of his/her operations in the remote scene before making new action [5]. Teleoperation data transmission delay can be ranging from milliseconds to several minutes, depending on distance and communication medium. In the control design, this latency imposes a tradeoff between the requirements of stability and performance [6]. In Vehicle indirect visual feedback, teleoperation is subjected to many sensors problems, some of these are:

1) Time lag: In video transfer feed and control commands.

2) Loss of situational awareness: Control inputs and vehicle reaction are not synchronized.

3) Limited field of view (FOV): Cameras mounted on the vehicle have limited field of view.

4) Sensor fusion and synchronization: Fusing many sensors is a challenge [7].

Sensors in teleoperated robotic systems are used to give complete coverage, such as a ring of ultrasonic sensors around a robot and to provide complementary information, such as cameras and Lidars. The task of sensor fusion is combining sensor data into a usable form for the operator [8].

\section{TIME-Delay COMPENSATION AlgORITHM}

In this work, the sensor used for teleoperation is the Laser Range finder; which is used for real-time 2D environment construction and for relative position estimation by range detection. The advantages from using the Laser scanner data are its accuracy and its relatively small size data compared to other sensors. Lidar is mounted on the vehicle at height $195 \times 10^{-3} \mathrm{~m}$ to detect the roads and obstacles in front of the vehicle. The sensing system provides a $270^{\circ}$ horizontal field of view in front of the vehicle with the distances up to $80 \mathrm{~m}$ as shown in Fig. 1(a). Additionally, the DGPS/INS provides precise vehicle localization, position and orientation [2] and [9].

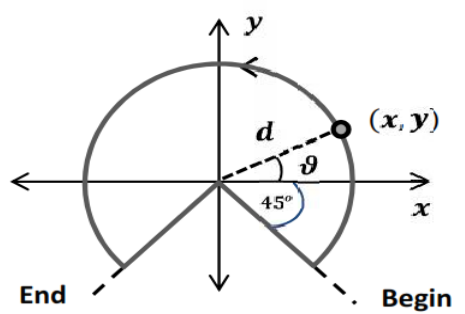

(a) Lidar scan plane.

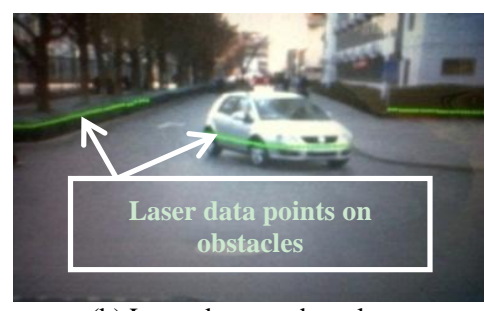

(b) Laser data on obstacles.

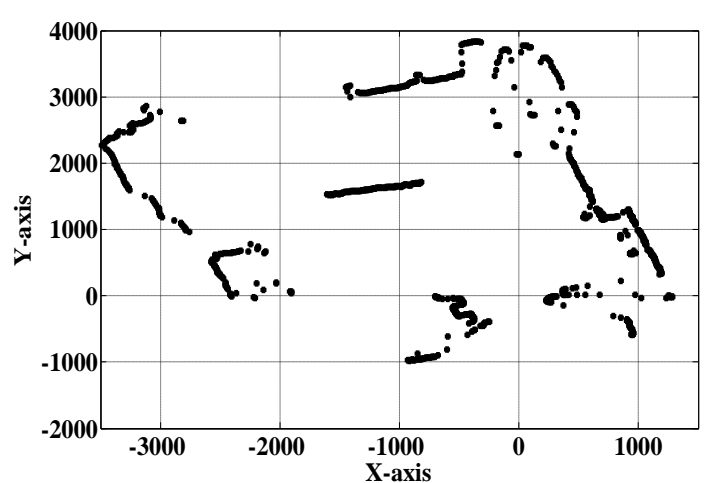

(c) Incoming Laser data.

Fig. 1. Laser scanner data experiment in BIT campus.

\section{A. Laser Scanner Data w. r. t. the Vehicle Current Position $t$}

In BIT campus, we used the Lidar data for the environment construction experiment and the laser points are plotted [10] using Mat-Lab codes as in Fig. 1(c); 1081 points, with angle range $\left[-45^{\circ}, 225^{\circ}\right]$ and with angular resolution $0.25^{\circ}$ and the distance unit is millimeter. For sharing information between computers and sensors, RS-232 and RS-422 protocols are adopted. Ethernet link is used for computers communication, and vehicle control ECUs are connected via CAN bus [11].

\section{B. Predicting the Vehicle Position in the Future Time}

In our mechanism, we proposed a solution to the latency in data transfer by predicting the vehicle position in the future time. That is to say, we let the operator see the vehicle in the predicted position after time $\Delta t$ in the future when the control command will arrive at, and be acted upon, by the vehicle.

We first predict the vehicle position in this future time and this predicted position is monitored to the operator in GCS; this time is equal to the time-delay computed in BIT laboratory which is less than $50 \times 10^{-3} \mathrm{~s}$. The predicted vehicle displayed is virtual one so it is quite simple to predict the vehicle in any position in the future. In these calculations, we choose this future time value to be $50 \times 10^{-3} \mathrm{~s}$ which is slightly larger than the value of the time-delay calculated. In this case the real vehicle can make up some of the prediction errors before they happen. This is accomplished as we consider the virtual vehicle is a leader one and the real one follows it, as the path followed by the virtual vehicle is sent to the real one as the command input [12].

We consider the real vehicle position in the origin coordinates $(0,0)$ w.r.t. vehicle frameand the virtual vehicle will be predicted after time $\Delta t$ in the future in $\left(x_{2}, y_{2}\right)$ coordinates in any place in front the vehicle and the software of the experiment permits to predict the virtual vehicle in any place by giving the program the coordinates $\left(x_{2}, y_{2}\right)$ in millimeter. And the distance $C$ from the vehicle current position to its predicted position which can be easily computed as shown in Fig. 2 and it is very small so the errors in the position and heading angle will be corrected by the real vehicle when it moves to follow the predicted one [13].

\section{Obstacles Position w. r. t. the Predicted Position}

For the environment construction with time-delay compensation we have to calculate, when the vehicle is in the current time, the obstacles position w. r. t. the vehicle 
predicted position. In this algorithm, what we seek is to calculate the Laser data for the predicted vehicle position at time $t+\Delta t$ using the available data in the current timet. These Laser scanner data are the range distance $d_{2}$ and the range angle $\vartheta_{2}$ for each laser point as in Fig. 2.

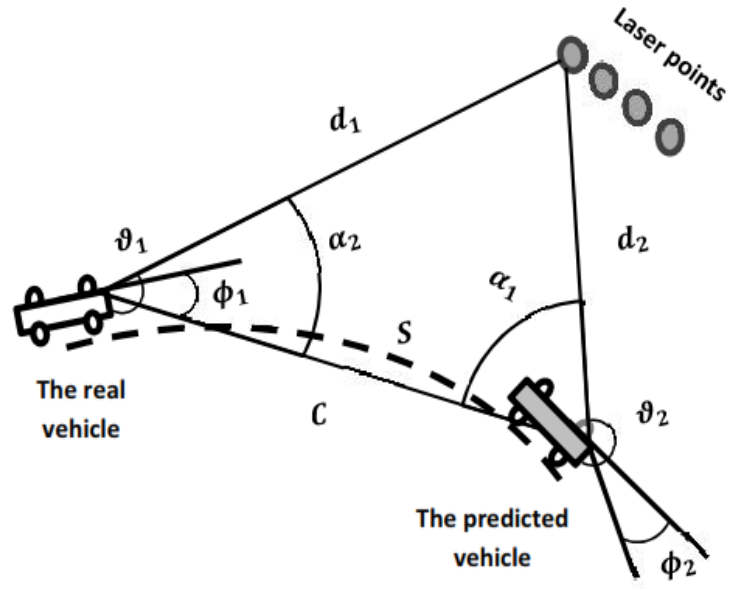

Fig. 2. Laser data w. r. t. the predicted position.

It is seen that the virtual triangle whose vertices are: the vehicle current position, the vehicle predicted position and Laser point on an obstacle; also, the proposed angles $\alpha_{1}$ and $\alpha_{2}$ are opposite to range distance of the laser point w. r. t. the real vehicle $d_{1}$ and the range distance of the same laser point w. r. t. the predicted vehicle $d_{2}$ respectively, and by simple calculations we can find the Laser range finder data w. r. t. the predicted position when the vehicle is in the current time as follows:

$$
\left.\begin{array}{c}
\alpha_{2}=\vartheta_{1}-\phi_{1}-90^{\circ} \\
d_{2}=\sqrt{d_{1}^{2}+C^{2}-2 d_{1} C \cos \left(\alpha_{2}\right)}, \\
\vartheta_{2}=270^{\circ}-\sin ^{-1}\left(d_{1} \sin \alpha_{2} / d_{2}\right) .
\end{array}\right\}
$$

At this step, the environment surrounding the vehicle in the predicted position is constructed. At this future time, the control signals will arrive and will be executed by the real vehicle, at the time before the real vehicle arrives to the predicted position [12]. In the GCS, the operator is driving the virtual vehicle and the path followed by the virtual vehicle is sent to the real vehicle as the commands inputs [14] By this way, the real vehicle is driven along the path designed by the operator using the virtual vehicle [15]. In this mechanism, the system produces a continuously predictive display that appears to operate in real time compensating time-delay as in Fig. 3.

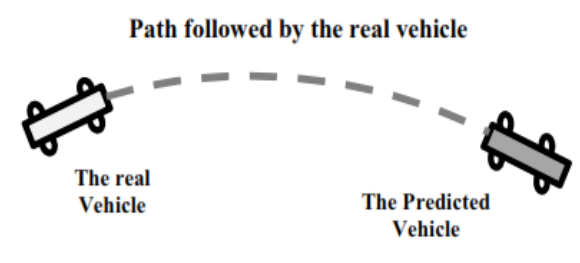

Fig. 3. The real vehicle follows the predicted one.

\section{EXPERIMENTAL RESULTS}

The algorithm simulation results show that the environment is continuously available in real time and the operator feels as if he/she controls the real vehicle directly with no latency achieving telepresence as illustrated in the steps of the detailed algorithm in Fig. 4. Moreover, the experiments results for the environment construction compensating time-delay using Laser range finder are shown in Fig. 5, whereas the black and grey points are the environment constructed using the laser data points w. r. t. the vehicle current and predicted positions respectively which are both available to the operator in the GCS when the vehicle is in the current time. It is shown in Fig. 5 (c) the laser data w. r. t. to the predicted vehicle only which will be available to the operator screen in GCS in the current time. And, the operator remotely drives the virtual predicted vehicle and these teleoperation commands which the operator performs are sent to the real vehicle as control commands. When these commands arrive to the real vehicle after time-delay $\Delta t$ the real vehicle reaches to the position before the predicted position mentioned above and it is obliged to follow the path of the virtual vehicle and thus eliminating any prediction errors before they happen [16], [17]. Hence, the operator has the sensation that the real vehicle is being controlled and teleoperated with no latency which achieves telepresence.

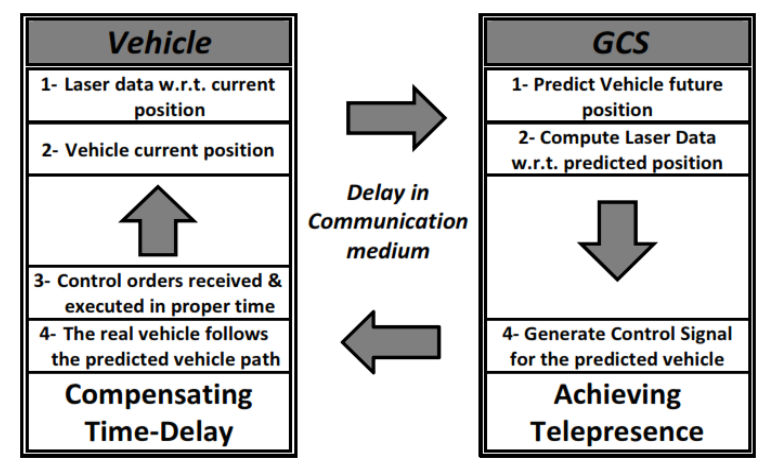

Fig. 4. BIT teleoperation algorithm using Laser scanner.

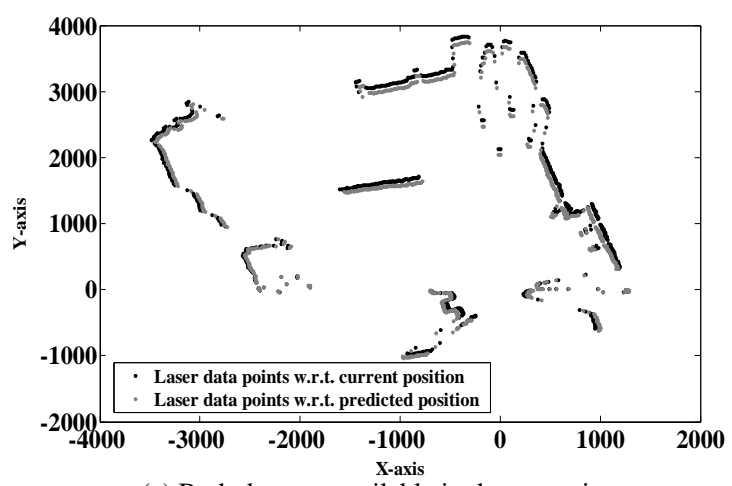

(a) Both data are available in the same time.

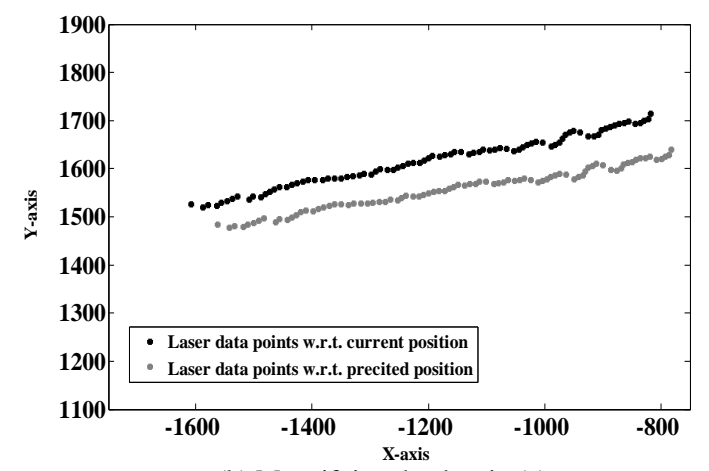

(b) Magnifying the data in (a). 


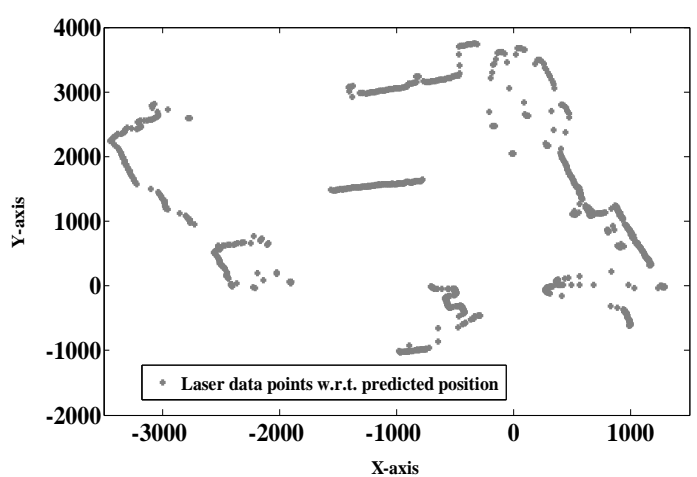

(c) Laser data w. r. t. predicted positions only.

Fig. 5. Laser scanner data w. r. t. current and predicted positions.

\section{CONCLUSION AND FUtURE WORK}

This paper has proposed a solution to the latency in environment construction using Laser range finder which is considered as a part of the work done in modifying the BIT $\mathrm{AGV}$ in order to be remotely controlled for the teleoperation purpose. In this approach, we construct the environment using the laser scanner with a predefined time offset $\Delta t$. This offset causes the laser scanner to construct the environment ahead of the current time, such that this constructed environment is always and readily available at the time it is supposed that the vehicle is driven in. This offset time is calculated such that it is the time required for transmitting sensor data to the GCS and transmitting the control signals from the GCS to the vehicle. The experiments show very good results in performance and transparency since the Teleoperation environment w. r. t. to the predicted position is available for the operator in the current time compensating latency. And also, the simulation results of the algorithm show that it is applicable for short and long time-delays.

The future work, we will be incorporating on the dynamic environment problems specially the high speed obstacles and integrating the algorithm in the BIT AGV control system to validate its efficiency. Also, we are looking for examining the algorithm using the Velodyne (3D Laser scanner) data for time-delay compensation using 3D environment construction and using also a CCD camera for coloring the laser data by the RGB color model on the camera images.

\section{ACKNOWLEDGMENT}

All the authors would like to thank the National Natural Science Foundation of China for partly supporting this work (No. 90920304 and 91120015).

Also, one of us (H. Nasry) would like to thank the members of intelligent vehicle research center in Beijing Institute of Technology for their great help and effort to support this work.

\section{REFERENCES}

[1] P. F. Hokayem and M. W. Spong, "Bilateral teleoperation: An historical survey," Automatica, vol. 42, pp. 2035-2057, Dec. 2006.

[2] Q. Chen, O. Ozguner, and K. Redmill, "Ohio State University at the 2004 DARPA grand challenge: Developing a completely autonomous vehicle," Intelligent Systems IEEE, vol. 19, no. 5, pp. 8-11, Sept.-Oct. 2004.
[3] S. Sirouspour and A. Shahdi, "Adjustable autonomy for mobile teleoperation of personal service robots," in Proc. RO-MAN, 2012 IEEE, pp. 933-940, Sept. 9-13, 2012.

[4] R. J. Anderson and M. W. Spong, "Bilateral control of teleoperators with time delay," IEEE Trans. on Automatic Control, vol. 34, no. 5, pp. 494-501, May 1989.

[5] P. Arcara and C. Melchiorri, "Control schemes for teleoperation with time delay: A comparative study," Robotics and Autonomous Systems, vol. 38, no. 1, pp. 49-64, Jan. 2002.

[6] M. Franken, S. Stramigioli, R. Reilink, C. Secchi, and A. Macchelli, "Bridging the gap between passivity and transparency," Robotics, Science and Systems, 2009.

[7] T. Fong, C. Thorpe, and C. Baur, "Advanced interfaces for vehicle teleoperation: Collaborative control, sensor fusion displays, and remote driving tools," Autonomous Robots, vol. 11, no. 1, pp. 77-85, July 2001.

[8] M. S. Kadavasal and J. H. Oliver, "Sensor augmented virtual reality based teleoperation using mixed autonomy," J. Comput. Inf. Sci. Eng. vol. 9, no. 1, March 2009.

[9] W. K. Wei, "Integration of GPS and DR navigation systems," in Proc. Vehicle Navigation and Information Systems Conference, vol. 2, pp. 635-643, Oct. 20-23, 1991.

[10] Q. L. Zhang and R. Pless, "Extrinsic calibration of a camera and laser range finder," in Proc. International Conference on Intelligent Robots and Systems, vol. 3, pp. 2301-2306, 28 Sept.-2 Oct. 2004.

[11] X. J. Zhao, H. O. Liu, Y. M. Man, K. Liu, W. D. Deng, H. Y. Chen, J. W. Gong, and G. M. Xiong, "Developing a fully autonomous ground vehicle," IST Transactions of Robotics, Automation and Mechatronics, 2010.

[12] A. Kelly, E. Capstick, D. Huber, H. Herman, P. Rander, and R.Warner, "Real-Time photorealistic virtualized reality interface for remote mobile robot control," in Proc. The 14th ISRR, pp. 211-226, Aug. 31-Sept. 3, 2009

[13] C. Scrapper, A. Takeuchi, T. Chang, T. Hong, and M. Shneier, "Using a priori data for prediction and object recognition in an autonomous mobile vehicle," SPIE, vol. 5083, pp. 414-418, Sept. 2003.

[14] X. L. Jin, X. J. Zhao, J. W. Gong, and Y. Jiang, "Design of a longitudinal controller for intelligent vehicle based on Fuzzy-PID," in Proc. IEEE International Conference on Vehicular Electronics and Safety, pp. 269 - 273, 10-12 July, 2011.

[15] H. Nasry, J. W. Gong, G. M. Xiong, Y. Jiang, and H. Y. Chen, "A novel predictive control for teleoperation with time-delay," in Proc. 2012 International Conference on Electrical Engineering and Computer Science, August, 2012.

[16] X. J. Zhao and H. Y. Chen, "Analysis and control of automatic steering system," in Proc. 2010 International Conference on Measuring Technology and Mechatronics Automation, vol. 1, pp. 529-534, 13-14 March, 2010.

[17] S. Sirouspour and A. Shahdi, "Model predictive control for transparent teleoperation under communication time delay," IEEE Trans. on Robotics, vol. 22, no. 6, pp. 1131-1145, Dec. 2006.

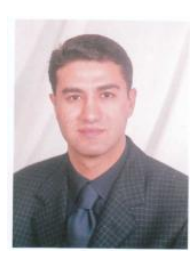

Hany Nasry was born in Giza, Egypt, on August 8. 1974 $\mathrm{He}$ received his B.E. degree in Electrical and Communications Engineering from Military Technical College, Cairo, Egypt in 1997, and M.S. degree in Mathematics from Military Technical College, Cairo, Egypt in 2003, and the M.S. degrees in Geometric Algebra applications in guidance and control systems. He has been member of Military Technical College in the Mathematics Department since1999till 2010.Currently he is pursuing his $\mathrm{Ph} . \mathrm{D}$. degree in Mechatronics and Teleoperation at Beijing Institute of Technology, Beijing, China. He published: "Application of Clifford Algebra to Navigation", in Sci. Bult. Fac. Eng. A in Shams University, vol. 39(4), December 2004,"Application Of Clifford Algebra To Kinematics" in Proc. 13th International Conference On ASAT, Military Technical College, Egypt, May 2008, and "A Novel Predictive Control for Teleoperation with Time-Delay", in Proc. 2012 International Conference on Electrical Engineering and Computer Science (EECS2012), Shanghai, China, August, 2012.His research interests are in the areas of sensors, intelligent vehicles, robotics, Geometric Algebra and control systems. Hany Nasry is a Ph.D. student member of the Intelligent Vehicle Research Center at the School of Mechanical and Vehicular Engineering, Beijing Institute of Technology, Beijing, China. 


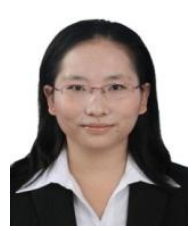

Ye Chun-Lan was born in YunNan, China, on September, 1988. She received her B.E. degree in Computer Science and Technology from Beijing Institute of Technology, Beijing, China in 2011. Currently she is pursuing her M.S degree in Vehicle Engineering at Beijing Institute of Technology, Beijing, China. Her research interest covers multi-sensor data fusion and 3D environment reconstruction for intelligent vehicle. Ye Chun-Lan is a student member of the Intelligent Vehicle Research Center, Beijing Institute of Technology, Beijing, China.

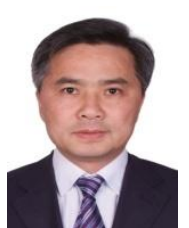

Gong Jian-Wei was born in Hunan, China, on April. 1969. He received his B.E. degree in Mechanical Engineering from National University of Defense Technology of China, China in 1992, and Ph.D. degree in Mechanical and Electronic Engineering from Beijing Institute of Technology, Beijing, China in 2002. Currently he is an Associate Professor in the school of Mechanical engineering at Beijing Institute of Technology, Beijing, China. He published: "Developing a Fully Autonomous Ground Vehicle," IST Transactions of Robotics, Automation and Mechatronics 2010, "Design of a Longitudinal Controller for Intelligent Vehicle Based on Fuzzy-PID," in Proc. IEEE International Conference on Vehicular Electronics and Safety, pp. 269 - 273 , Beijing, China, 10-12 July, 2011. His research interests are in the areas of robotics, vehicles and intelligent vehicle. His research interests are in the areas of intelligent vehicle technology and autonomous vehicles. Assoc. Professor Gong Jian-Wei is a member of the intelligent vehicle research center at the School of Mechanical and Vehicular Engineering, Beijing Institute of Technology, Beijing, China.

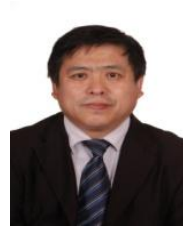

Chen Hui-Yan was born in HeiLong Jiang, China, on March21. 1961. He received his B.E. degree in Electrical Engineering from HeiLong Jiang University, China, and Ph.D. degree in Vehicular Engineering from Beijing Institute of Technology, Beijing, China in 2004. Currently he is a Professor in the school of Mechanical engineering at Beijing Institute of Technology, Beijing, China. He published: "Developing a Fully Autonomous Ground Vehicle," IST Transactions of Robotics, Automation and Mechatronics 2010 "Analysis and Control of Automatic Steering System," in Proc. 2010 International Conference on Measuring Technology and Mechatronics Automation, vol.1, pp. 529-534, 13-14 March, 2010. His research interests are in the areas of robotics, vehicles and intelligent vehicle. Professor Chen Hui-Yan is the head of the intelligent vehicle research center at the School of Mechanical and Vehicular Engineering, Beijing Institute of Technology, Beijing, China. 